\title{
A Three Dimensional Microflown
}

\author{
D.R. Yntema, J.W. van Honschoten, H.-E. de Bree, R.J. Wiegerink, M. Elwenspoek \\ Transducers Science and Technology group \\ MESA+ Institute for Nanotechnology, University of Twente, the Netherlands
}

\begin{abstract}
An integrated three dimensional acoustic particle velocity sensor is realized. The integration of multiple sensors on a single silicon die leads to improvements in terms of better a better reproducible sensor and a very small sensor to sensor distance allowing accurate single point measurements. Initial measurements performed show that three dimensional noise source finding is possible with this sensor.
\end{abstract}

\section{INTRODUCTION}

Acoustic sound fields consist of a combination of pressure change and particle velocity. While acoustic pressure is a scalar quantity, particle velocity has both magnitude and direction, making it a vector quantity. Therefore, to measure the complete particle velocity field instantaneously a threedimensional sensor is needed. So far, this has been done by mounting individually mounted Microflown sensors together in a single package [1],[2]. In this paper we present a monolithically integrated 3D sensor consisting of four sensors integrated on one silicon die. This will result in very good sensor reproducibility in terms of directivity (since sensor location and direction of sensitivity is now determined by lithography instead of manual mounting sensors together), easier mounting and a very small sensor to sensor distance.

\section{DESIGN}

Figure 1 shows a photograph of the realized sensor. Instead of integrating 3 sensors perpendicular to each other we chose to integrate 4 sensors at angles of 45 degrees with the chip surface. In Cartesian coordinates this corresponds to

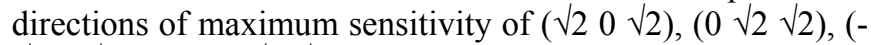
$\sqrt{2} \quad 0 \sqrt{2})$ and $(0-\sqrt{2} \sqrt{2})$.

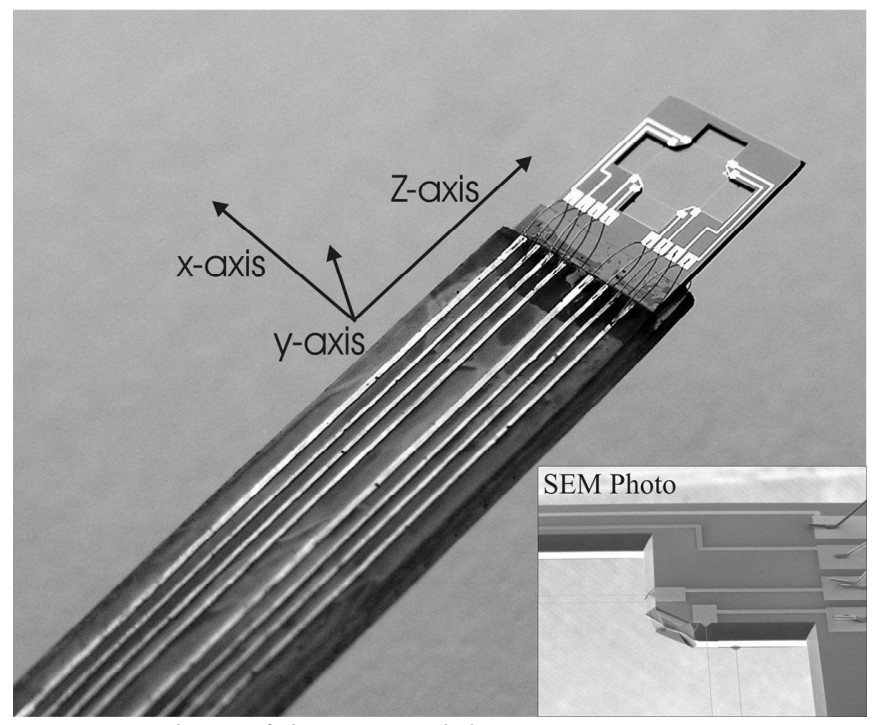

Figure 1, photo of the mounted device
This configuration has some interesting properties; firstly modeling is more reliable due to the symmetry and secondly it provides the possibility of calculating cross spectra between two signals in any direction. Taking the averaged cross spectrum between two signals provides less (uncorrelated) sensor noise thus allowing to measure lower particle velocity levels [3]. The four-sensor configuration provides at least two signals in any direction [4]. Due to the integration in one die the sensor can be extremely flat ( $250 \mu \mathrm{m}$ thick), this can be of benefit when measuring close to surfaces. Seen in the $\mathrm{Y}$ direction the sensor is of size $5 \mathrm{~mm} * 5 \mathrm{~mm} * 0.25 \mathrm{~mm}$, which is not smaller in surface as existing 3D microflown sensors, but the sensors are more closely spaced (max. $2.5 \mathrm{~mm}$ ) to each other. This allows almost single point particle velocity measurement, which can useful when measuring close to very small sources or at high frequencies

\section{FABRICATION}

Special $250 \mu \mathrm{m}$ thick double-side polished wafers are used because the wafer thickness defines the distance between the sensor wires in this design. To obtain a sensitivity direction of 45 degrees to the wafer surface the sensor wires are displaced by $250 \mu \mathrm{m}$ in relation to each other. In this way the distance between the wires becomes $354 \mu \mathrm{m}$.

Fabrication is done on both sides of the wafer to be able to work "out of plane", which is necessary for realization of the wanted sensitivity directions. Figure 2 shows a summary of the fabrication process.

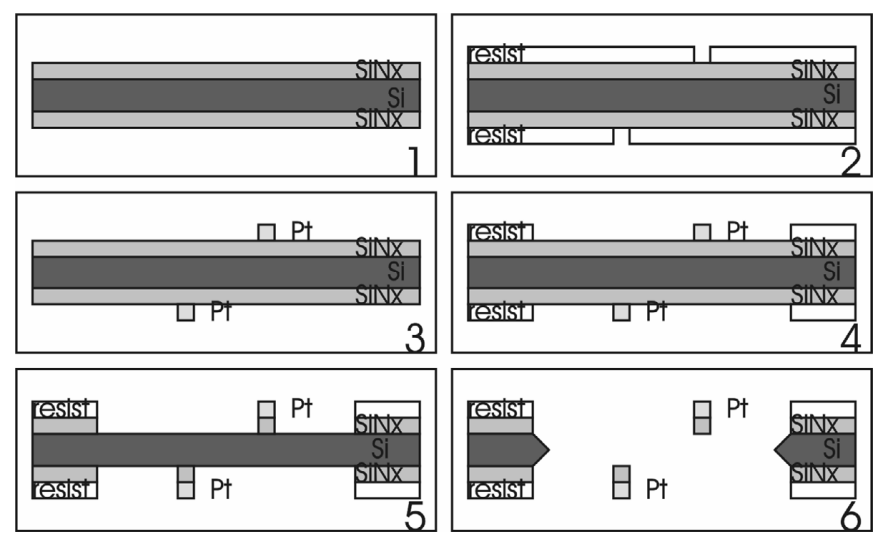

Figure 2, fabrication process

An insulating and construction layer of silicon nitride is deposited by LPCVD. Next a layer of platinum is deposited on patterned photo resist (figure 2,2). After lift-off of the photo resist the platinum will remain. Next a new layer of photo resist on top of the silicon nitride is patterned and the exposed silicon nitride will be etched by RIE, leaving an etching mask for $\mathrm{KOH}$ etching (figure 2,5). After etching of 
the silicon in $\mathrm{KOH}$ the sensor wires will be freed. The complete sensor is etched free from the wafer except for four small silicon beams holding the sensor in place; this provides a "break out" sensor, since dicing is almost certainly impossible without destructing the sensor wires.

For using the element it is mounted on a carrier printed circuit board, which again is mounted in a small tube for easier handling. For more frequent use the sensor must be packaged further since the constructed sensor is still very fragile.

Every pair of sensor wires is connected in series and powered with a low noise voltage supply; the series tap signal is amplified with a low noise amplifier to obtain a reasonable signal for further processing. All four channels are then measured simultaneously.

Sensor yield is approximately $25 \%$ with this process; on one standard 4" wafer approximately 20 working pieces can be made. By far most defects occur because of sensor wire breakage. With thicker sensor wires the yield can be increased significantly; however this will decrease sensor performance at higher frequencies. When looking closely at the wires it can be seen that the wires are not smooth at the edges and that wire breakage simply occurs due to bad patterning of the platinum layer. Possibly with another type of resist like image reversal resist the patterning of the platinum wire is more accurate. The wire thickness will then be more constant over its length and this will decrease sensor failure.

Furthermore in this design the inner, protruding edges of the etched silicon are etched significantly (see figure 1, SEM-photo), however not too much to destroy the wire supports. The amount of etching was controlled by using sacrificial structures at these edges. A too large sacrificial structure could result in sensor wire breakage due to remains of silicon nitride (the remaining $\mathrm{KOH}$ mask) colliding with the wires.

To keep a slight tension on the wires to prevent phenomena like wire resonance effects and to provide a better support small triangular shaped end points are used. The triangular shapes tend to bend to the inner of the wafer. Also the temperature distribution at the edges due to this will be more gradual. As can be seen in figure 1, SEM Photo, the wires are on both sides of the wafer and the triangular shapes are visible at each end of the sensor wires.

\section{SIMULATION AND MEASUREMENT RESULTS}

The global behavior of the sensor was analyzed using finite element simulations. The simulations show that a local deviation from the applied particle velocity direction of 5 degrees occurs at $1.7 \mathrm{kHz}$ (see Figure 3).

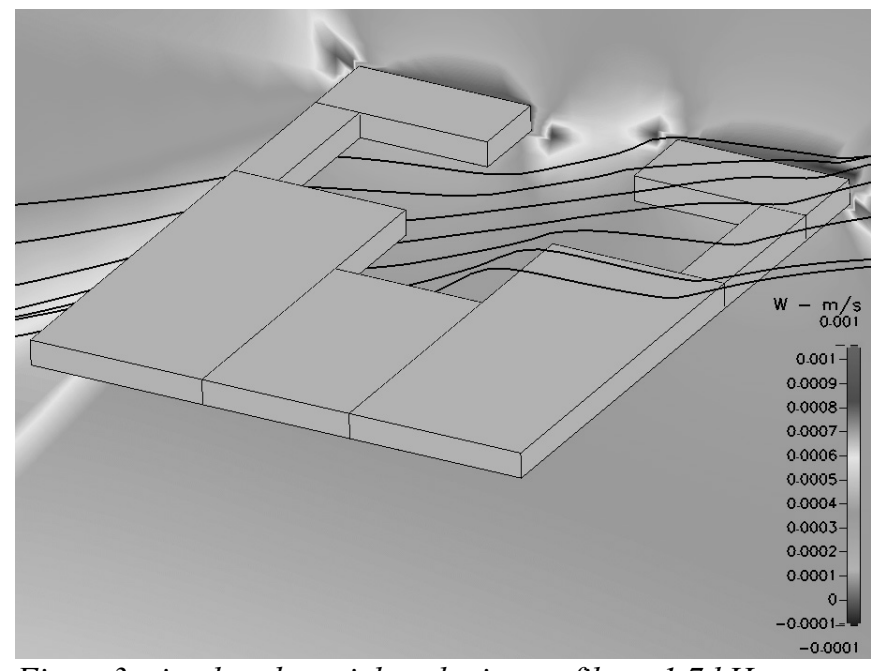

Figure3, simulated particle velocity profile at 1,7 kHz

Initial measurements were performed at a frequency of $1.7 \mathrm{kHz}$ using a measurement setup that allowed rotation of the sensor along the two symmetry axes, namely the $\mathrm{x}$ axis and $\mathrm{z}$-axis (see figure 1 for orientation). The result of the measurement with rotation around the $\mathrm{x}$ axis is shown in Figure 5.

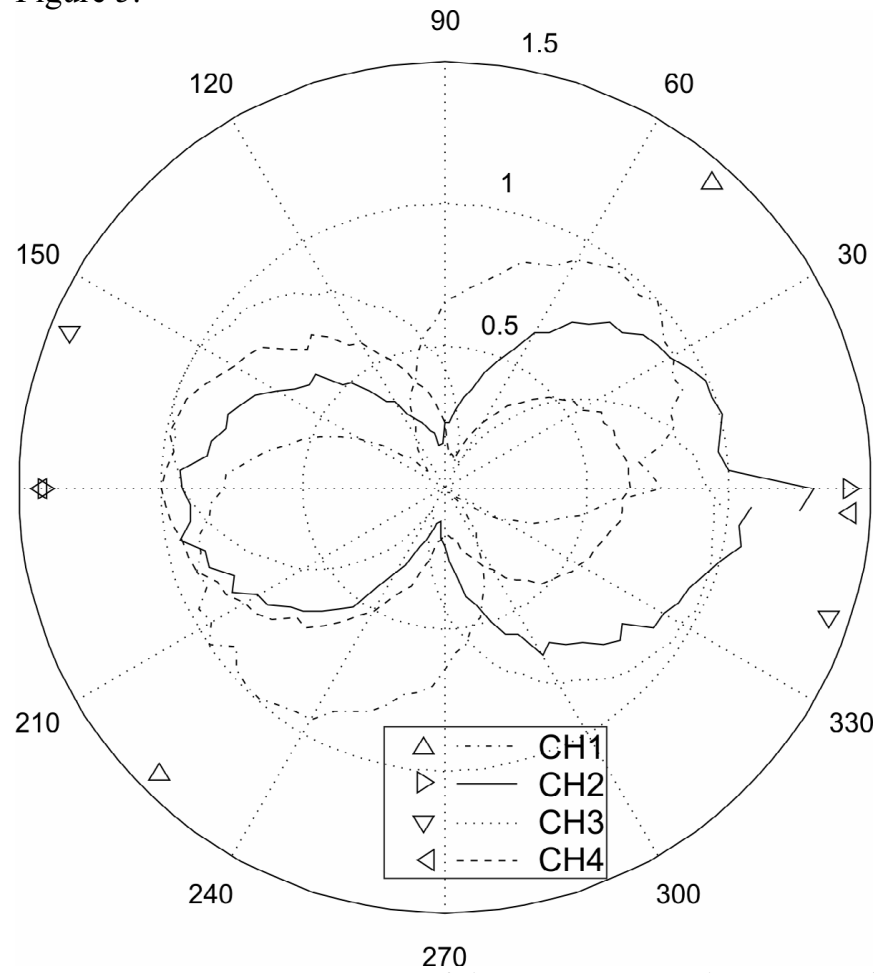

Figure 5, Sensitivity pattern of the sensors at $1.7 \mathrm{kHz}$, rotated along the $x$-axis. Triangles show the measured sensitivity directions. Channel 2 and 4 should have directions of 0 and 180, ch1 at 45 and 225, ch3 at 135 and 315 degrees.

There is clear figure-of-eight (or vector) response for each sensor, but the expected direction of sensitivity is not matching the measured direction perfectly. Channel 1, 2 and 4 are aligned perfectly, but channel 3 is at least ten degrees off. This is very likely the result of the package around the sensor. Sensor 3 is the sensor nearest to the mounting PCB. 


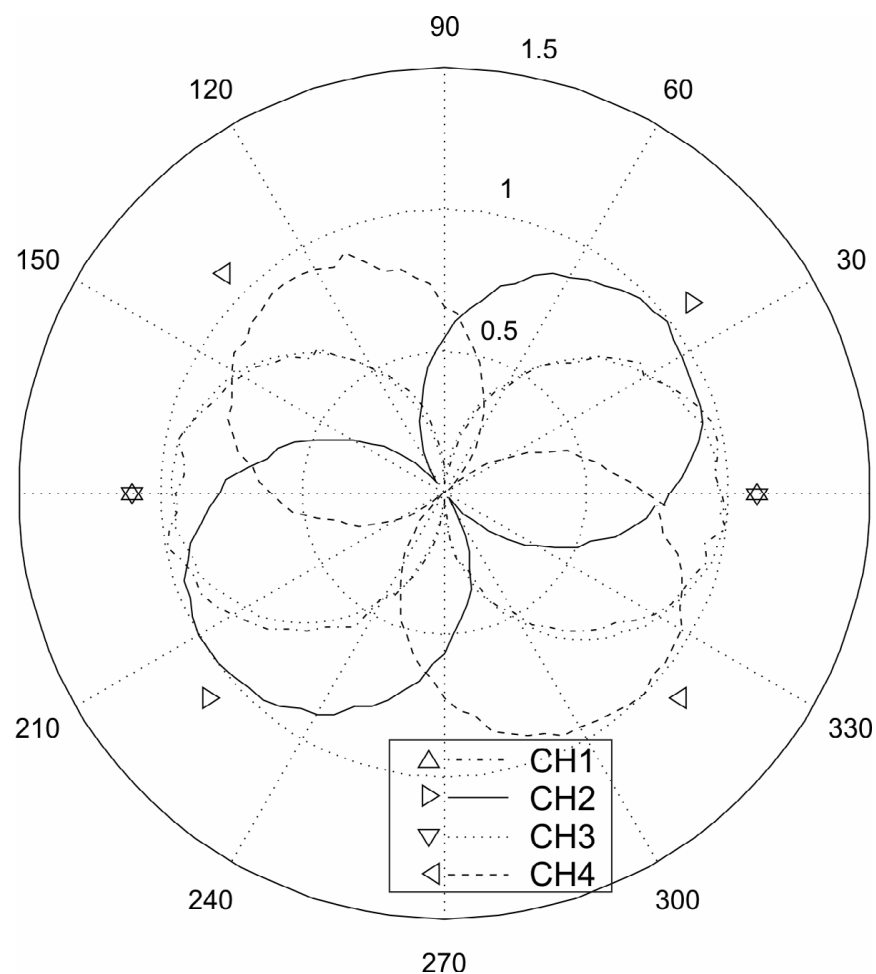

Figure 6, Sensitivity pattern of the sensors at $1.7 \mathrm{kHz}$, rotated along the z-axis. Triangles show the measured sensitivity directions. All channels should have a 45 degrees angle with the $x$ or $y$ axis.

An analytical solution to this problem is not readily available. However simulations are predicting this effect.

Measurements of frequency response and sensitivity level reveal that the sensors are quite identical with less than $6 \mathrm{~dB}$ difference (figure 7). The graphs are showing the transfer function between a reference pressure microphone and the device inside a tube. The peaks are due to standing waves inside the tube and give a calibration value of the sensor at the respective frequency [2].

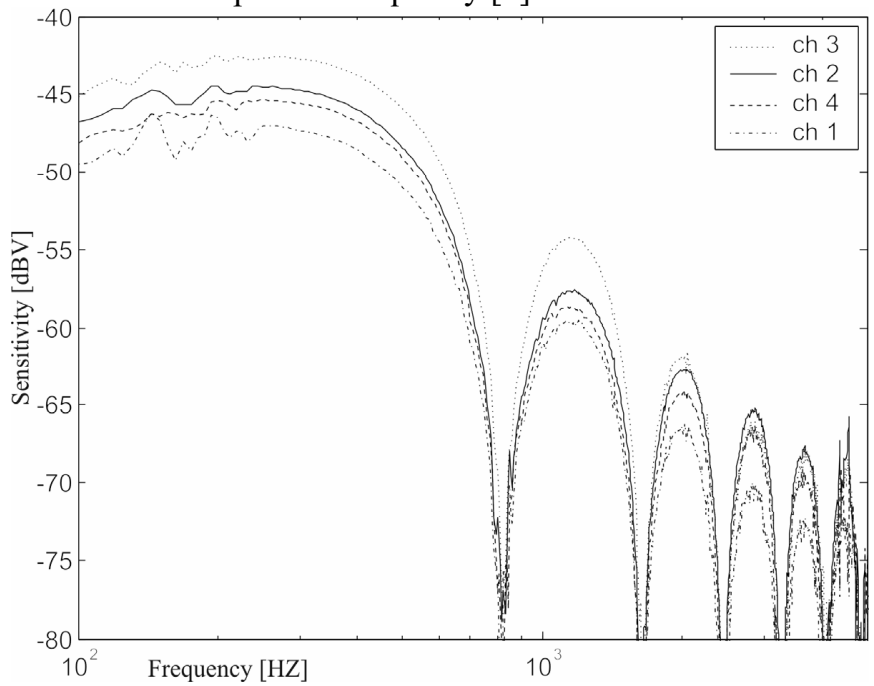

Figure 7, sensitivity levels of each sensor

Sensitivity levels are shown with pre-amplifiers connected. The sensitivity difference is partly due to the inequality of the sensor wire pairs; the resistance varies between 7 and $10 \mathrm{kOhm}$ per sensor pair. Clearly the sensor nearest to the mounting PCB has by far the largest sensitivity level, and this is most probably due to the particle velocity disturbance near the relatively large obstacle. Measurements are performed at a sensor voltage of 10 volts ( 5 volts per sensor wire) and a current of approximately $1.3 \mathrm{~mA}$ (depending on the sensor). This gives a "hot" resistance of $7.7 \mathrm{kOhm}$ and a power consumption of $1.3 \mathrm{mWatt}$ per sensor. This can be increased to approximately $6 \mathrm{~mW}$ atts before the sensor ceases working. When measuring the sensitivity and the electrical noise the equivalent acoustic noise can be found by dividing sensitivity by the electrical noise. In figure 8 the results on the self noise of this sensor are shown. The sensitivity measured earlier (figure 7) is divided by the electrical noise. Peaks are due to the calibration method used. The scaling is adjusted so that $0 \mathrm{~dB}$ is equivalent to $0 \mathrm{~dB}$ acoustic pressure level in free field; $0 \mathrm{~dB}$ particle velocity is equivalent to $50 \mathrm{~nm} / \mathrm{sec}$. A fitted line shows the self noise for each power level. An optimal self noise level is found at $4 \mathrm{mWatt}$ (at 20 volts and $2 \mathrm{~mA}$ ).

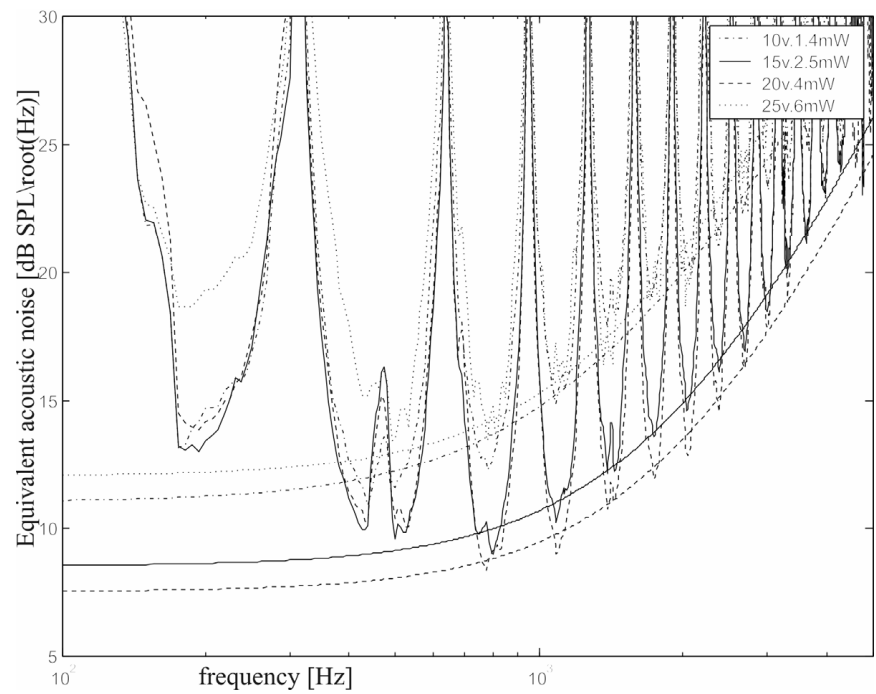

Figure 8, equivalent acoustic noise level; fitted lines show interpolated self noise levels.

\section{AN APPLICATION: NOISE SOURCE FINDING}

One of the possible applications of this sensor is noise source localization. With the four sensors combined it is possible to calculate the three dimensional particle velocity vector, and so the source of this particle velocity can be found. This can be done by simply using the power spectral density of each signal, but a better result can be attained when calculating the cross correlation signal between two channels. This results in a lower noise level because uncorrelated sensor noise will be averaged out [3].

When calculating the cross correlation between two sensors six different cross correlation signals can be obtained. From these signals the direction and magnitude of the applied particle velocity can be found. 
For a small set of different particle velocity directions measurements were performed. In a normal room of size $5 \mathrm{~m} * 5 \mathrm{~m} * 10 \mathrm{~m}$ a sound source at a distance of $0.5 \mathrm{~meter}$ is rotated around the sensor in the XZ plane (see figure 1) showing that in this experiment the direction of the particle velocity can be determined accurately within 7 degrees. In table 1 measurement results are given. With the particle velocity direction parallel to the $Z$ axis defined as zero degrees, the source is rotated in the $\mathrm{XZ}$ plane towards the $-\mathrm{X}$ and $+\mathrm{X}$ axis. Also the angle of the particle velocity and the $\mathrm{Y}$ axis can be found. Deviations are within 2 degrees here. Experiments with a larger component in the $\mathrm{Y}$ direction showed errors of the same order of magnitude as measurement results in the $\mathrm{XZ}$ plane.

For measurements on the particle velocity direction it is especially important that all sensors are calibrated with respect to each other; an absolute calibration value is of importance when the particle velocity level is also needed.

Table 1, noise source finding measurements

\begin{tabular}{|l|l|l|l|l|l|l|}
\hline $\begin{array}{l}\text { Angle of PV with } \\
\text { Z axis }\end{array}$ & -90 & -60 & -30 & 0 & 30 & 60 \\
\hline $\begin{array}{l}\text { Measured angle } \\
\text { of PV with Z axis } \\
\text { projected in XZ } \\
\text { plane }\end{array}$ & -90 & -67 & -34 & -7 & 34 & 64 \\
\hline $\begin{array}{l}\text { Measured angle } \\
\text { of PV with Y }\end{array}$ & -1 & 0 & 1 & 1 & 2 & 0 \\
\hline
\end{tabular}

\section{CONCLUSION}

A three dimensional particle velocity sensor element with four sensor elements has been made. Although measurements were satisfying and proved that the sensor was directional in the expected directions a better result can be obtained by redesigning the sensor. An even smaller outline will decrease deviations from the wanted sensitivity directions. By using finite element simulations the effect can be predicted on forehand and so the orientation of the wires can be adjusted on forehand so that the sensor is sensitive in the wanted directions.

Also different techniques for gaining a more constant sensor wire thickness can be applied to increase sensor stability and total yield. Measurements show that the sensor can be used successfully for noise source localization.

\section{REFERENCES}

[1] H-E. de Bree et al., The Microflown: a novel device measuring acoustical flows, Sensors and Actuators A 54 (1996), 552-557.

[2] H.-E. de Bree, "The Microflown: An acoustic particle velocity sensor," Acoustics Australia 31, 91-94 (2003)

[3] J.W. van Honschoten, W.F. Druyvesteyn, H. Kuipers, R.Raangs, G.J.M. Krijnen, Acta Acustica 90, 349355, 2004.

[4] D.R. Yntema and W.F. Druyvesteyn., "A four particle velocity sensor device" accepted for publication in JASA 2006 